\title{
Transmission of PAM4 Signals in ICXT-impaired Intra-datacenter Connections with PAM2 Signal Interference
}

\author{
Inês C. Jorge ${ }^{2}$, João L. Rebola ${ }^{1,2}$ and Adolfo V. T. Cartaxo ${ }^{1,2}$ \\ ${ }^{1}$ Optical Communications and Photonics Group, Instituto de Telecomunicações, Lisboa, Portugal \\ ${ }^{2}$ Instituto Universitário de Lisboa (ISCTE-IUL), Lisboa, Portugal \\ $\{$ icjea, joao.rebola, adolfo.cartaxo\}@iscte-iul.pt
}

\begin{abstract}
Keywords: Bit Error Rate, Inter-core Crosstalk, Intra-datacenter Connections, Multicore Fiber, Outage Probability, PAM4.
Abstract: Traffic in datacenters has been significantly increasing over the last few years. As a result, it is necessary to scale the connections of the datacenters to accommodate such an increase of traffic. The solution considered in this work is to use four-level pulse amplitude modulation (PAM4) and weakly-coupled multicore fibers (MCFs) to support intra-datacenter connections. However, transmission in weakly-coupled MCFs may be significantly degraded by inter-core crosstalk (ICXT). In this work, the impact of ICXT on the performance of PAM4 transmission in short-haul direct-detection links is evaluated considering two-level PAM (PAM2) signals in the interfering cores. The ICXT impact on the performance of the tested core with PAM4 transmission is evaluated using the bit error rate (BER), outage probability (OP) and eye-pattern analysis. Comparing to PAM2 transmission in the tested core, a $7.6 \mathrm{~dB}$ lower ICXT level is required to achieve an acceptable OP for a reference BER of $3.8 \times 10^{-3}$ in the PAM4 link.
\end{abstract}

\section{INTRODUCTION}

Datacenters provide an infrastructure to Internet online services such as web-browsing, e-mail, videostreaming, storage and file sharing, cloud computing and mobile services. Due to all these services and the emergence of $5 \mathrm{G}$ and Internet of Things (IoT), traffic in datacenters has been dramatically increasing over the last few years (Cisco, 2018). With the growth of capacity in datacenters, data traffic, which was mainly transmitted only from external datacenters to servers, started to be transfered between servers inside the same datacenter or anothers nearby supported by optical fiber technology. One of the current solutions to scale the capacity of datacenter connections is the use of multiple wavelengths to carry wavelength division multiplexing (WDM) channels, each channel considering the transmission of on-off keying modulated signals, also known as two-level pulse amplitude modulation (PAM2) signals. In 2007, the first generation of intra-datacenter technology operated at $10 \mathrm{~Gb} / \mathrm{s}$ using a single wavelength channel, PAM2 modulation and direct-detection was released (Zhou et al., 2019). In 2010, the technology has evolved into a second generation that operated at a $40 \mathrm{~Gb} / \mathrm{s}$ aggregated bit rate with $4 \mathrm{WDM}$ channels (Zhou et al., 2019). The third generation, from 2014, operated at $100 \mathrm{~Gb} / \mathrm{s}$ with $4 \mathrm{WDM}$ channels, each one at $25 \mathrm{~Gb} / \mathrm{s}$ with PAM2 transmission (Zhou et al., 2019). In 2017, a generation of $400 \mathrm{~Gb} / \mathrm{s}$ datacenter connections emerged, employing the more bandwidth-efficient four-level pulse amplitude modulation (PAM4), doubled the WDM channels in use to 8 and the data rate in each channel from $25 \mathrm{~Gb} / \mathrm{s}$ to $50 \mathrm{~Gb} / \mathrm{s}$, leading to an aggregated rate of $400 \mathrm{~Gb} / \mathrm{s}$ (Zhou et al., 2019). The PAM4 format is foreseen as cost-effective and efficient enabler of $100 \mathrm{G}$ and $400 \mathrm{G}$ per channel in datacenter connections (Perin et al., 2018). So, in the future, it is expected that PAM4 supported systems can lead to data rates of $800 \mathrm{~Gb} / \mathrm{s}$ and 1.6 Tb/s in the datacenters connections (Zhou et al., 2019).

Datacenter connections can be classified into intra-datacenter connections, which have a range up to $10 \mathrm{~km}$ or inter-datacenter connections that have a range up to $100 \mathrm{~km}$ (Perin et al., 2018). Intra and inter-datacenter communication systems use typically intensity modulation at the transmitter and directdetection at the receiver, to ensure lower cost (Perin et al., 2018). Intra and inter-datacenter links have as design priorities low cost, power consumption and port density, and exhibit reduced propagation problems when compared to long-haul systems since nonlinear effects are usually insignificant at these short 
propagation distances (Perin et al., 2018).

The signal transmission in datacenters is usually supported by single-core single-mode fibers (SCSMF). Recent SC-SMF transmissions have achieved a transmission capacity of about $100 \mathrm{~Tb} / \mathrm{s}$ and a capacity-distance product over $100 \mathrm{~Pb} / \mathrm{s} \cdot \mathrm{km}$ relying on the use of coherent-detection allied with sophisticated digital signal processing (Saitoh and Shoichiro, 2016). However, datacenter connections are still exploring direct-detection due to its lower cost and simplicity, and such high transmission capacities cannot be met in these connections. For the sake of saving space between the racks inside datacenters, reducing fiber per area density, and to reduce inventory issues, multicore fibers (MCFs) are one of the most attractive technologies to overcome the use of SC-SMF and scale the transmission capacity in intradatacenter connections (Butler et al., 2017). Homogeneous weakly-coupled MCFs are a promise to expand these capacity limits. In these fibers, each core has similar properties, as geometry and refractive index, all the cores have the same propagation constant and can be used as independent transmission channels (Klaus et al., 2017). However, MCFs lead to inter-core crosstalk (ICXT), which may limit significantly the short-haul direct-detection datacenter connection performance or the link reach. The ICXT results from undesired field coupling between the cores of the MCF. The ICXT is a stochastic process that has a random time variation which can cause random high levels of ICXT in a short period of time (Alves et al., 2019), (Alves and Cartaxo, 2017). This random behavior of ICXT can cause the system shutdown or long time periods of outage and fluctuations of the bit error rate (BER). In this work, the impact of ICXT on the performance of PAM4 transmission in intradatacenter links impaired by PAM 2 transmission in the interfering cores is analyzed through several metrics, such as BER, eye-pattern analysis and outage probability (OP). This scenario will probably be encountered in the upgrade of a future existing intradatacenter link with PAM2 transmission in all cores of the MCF (Rebola et al., 2019c) to support PAM4 transmission in some cores.

\section{SIMULATION MODEL}

Fig. 1 shows the system model for the intra-datacenter link, which typically operates at a wavelength near $1310 \mathrm{~nm}$, to minimize total chromatic dispersion (CD). These links are usually unamplified, resulting in a low power margin (Perin et al., 2018).

The equivalent system model of the intra-

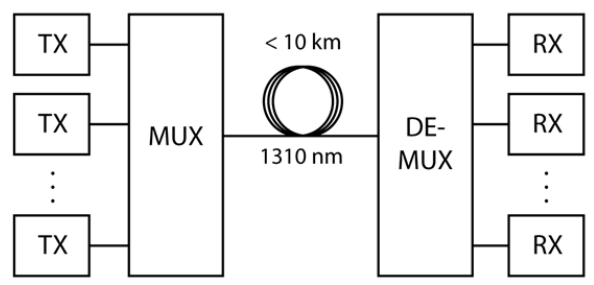

Figure 1: Typical intra-datacenter links. TX: transmitter, MUX: multiplexer, DEMUX: demultiplexer, RX: receiver.

datacenter link shown in Fig. 1, supported by MCFs with PAM signal generation at the transmitter is shown in Fig. 2. It is composed by two optical PAM transmitters, one for each fiber core. The tested core considers PAM4 transmission and the interfering core assumes PAM2 signalling. An ideal linearized model without chirp for each optical transmitter is assumed. The weakly-coupled MCF model considers an interfered core $n$ and a single interfering core $m$, and the signals are propagated in the two polarization directions $\mathbf{x}$ and $\mathbf{y}$ inside the MCF (Soeiro et al., 2017). The ICXT is described by the discrete changes model (DCM). At the output of the interfered core $n$, the ICXT induced by the core $m$ appears added to the signal transmitted in core $n$. The direct-detection optical receiver model includes the PIN photodetector, the electrical noise addition due to photodetector and electrical amplifier, electrical filtering and the decision circuit to decide on the transmitted PAM4 symbol. In the following subsections, detailed descriptions are presented to some of these models.

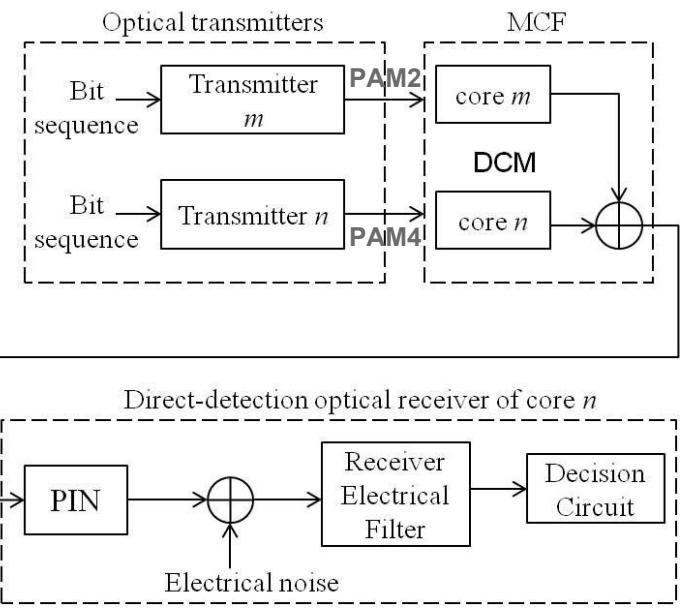

Figure 2: Equivalent system model.

\subsection{PAM4 Signal Characterization}

In this subsection, the generation and the characterization of the PAM4 signal of the tested core is explained. 


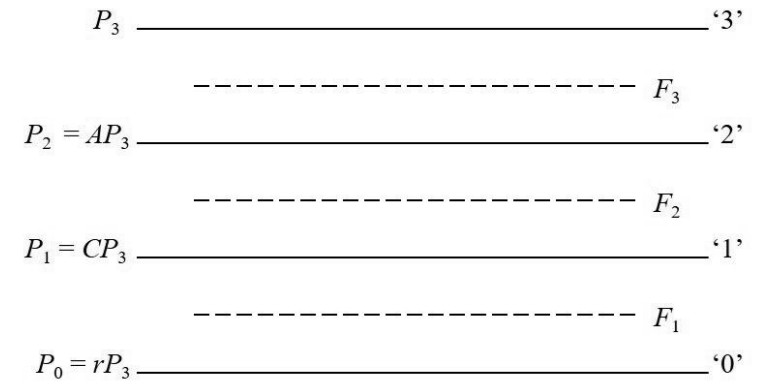

Figure 3: Representation of the power levels of an optical PAM4 signal with non-zero extinction ratio and the corresponding decision thresholds for an optical receiver with signal independent noise dominance.

The PAM4 modulation format maps two bits in each PAM4 symbol, which reduces the bandwidth requirement by half when compared to PAM2 signals (Lv et al., 2016). In the simulator, the PAM4 symbol sequence is generated using deBruijn sequences obtained from Galois arithmetic of maximum length $4^{N_{b}}$, where $N_{b}$ represents the number of positions of the offset register used to generate the sequence. In this way, a deBruijn sequence is generated where the PAM4 symbols ' 0 ', ' ${ }^{\prime} 1^{\prime}, 2^{\prime}$ and ${ }^{\prime} 3^{\prime}$ are equally likely to occur. Fig. 3 shows a representation of the power levels of an ideal PAM4 signal assuming a non-zero extinction ratio, where ' 0 ', ' 1 ' , ' 2 ' and ' 3 ' represent the symbols of the PAM4 signal; $F_{1}, F_{2}$ and $F_{3}$, are the ideal decision thresholds; $P_{3}, P_{2}, P_{1}$ and $P_{0}$ are the powers corresponding to each one of the symbols, and $r$ represents the extinction ratio defined as in (Rebola and Cartaxo, 2000).

$$
r=\frac{P_{0}}{P_{3}}
$$

Eq. 1 corresponds to the inverse of the definition of the extinction ratio as adopted by ITU-T (Telecommunications Standardization Section of ITU-T, 2009). The constants $A$ and $C$ define the spacing between the intermediate levels of the PAM4 signal. As the performance in intra-datacenter links is only affected by electrical noise, the PAM4 power levels should be equidistant (Rebola and Cartaxo, 2000). As such, $A$ and $C$ are given, respectively, by (Rebola and Cartaxo, 2000)

$$
\begin{aligned}
& A=\frac{2}{3}+\frac{1}{3} r \\
& C=\frac{1}{3}+\frac{2}{3} r
\end{aligned}
$$

The average power of the generated PAM4 optical signal is then given by

$$
\bar{P}_{a v}=\frac{1+A+C+r}{4} P_{3}
$$

These power levels are used to generate the PAM4 signal with rectangular-shaped pulses. Then, this ideal PAM4 signal is filtered by a $3^{\text {rd }}$ order Bessel filter, in order to have a more realistic pulse shape at the optical transmitter output. The $-3 \mathrm{~dB}$ bandwidth of the Bessel filter is equal to the symbol rate $R_{s}$. This filtering models the frequency limitations due to the electrical part of the PAM4 transmitter.

The PAM2 signal in the interfering core is generated as described in (Rebola et al., 2019c).

\subsection{Discrete Changes Model}

In this subsection, the simulation model used in this work, known as dual polarization DCM, to characterize the ICXT induced by the cores is presented (Soeiro et al., 2017). It should be highlighted that this model provides a very accurate characterization of the ICXT impact in direc-detection optical communication systems, as shown in (Alves et al., 2019), by comparison with experimental results.

Linear propagation along the MCF is assumed in the two cores, where $m$ and $n$ are the interfering and tested (interfered) cores, respectively. The dual polarization DCM considers the power splitting of the transmitted PAM signal by the polarization directions $\mathbf{x}$ and $\mathbf{y}$ at the input of the MCF to obtain the ICXT in both polarizations. The transfer functions $F_{x, x}(\omega)$ and $F_{y, x}(\omega)$ are used to obtain the ICXT generated in the polarization $\mathbf{x}$ of core $n$ from polarizations $\mathbf{x}$ and $\mathbf{y}$ of core $m$. The transfer functions $F_{x, y}(\omega)$ and $F_{y, y}(\omega)$ are used to obtain the ICXT generated in the polarization $\mathbf{y}$ of core $n$ from both polarizations of core $m$ (Soeiro et al., 2017). The transfer functions $F_{a, b}(\omega)$, model the frequency response of the ICXT from the polarization $\mathbf{a}$ at the input of core $m$ to the polarization $\mathbf{b}$ at the output of core $n$ and are given by (Soeiro et al., 2017)

$$
\begin{aligned}
& F_{a, b}(\omega)=-\frac{j}{\sqrt{2}} \bar{K}_{n m} \exp \left[-j \bar{\beta}_{n}(\omega) L\right] \\
& \sum_{k=1}^{N_{p}} \exp \left[-j\left(\bar{\beta}_{m}(\omega)-\bar{\beta}_{n}(\omega)\right) z_{k}\right] \exp \left[-j \phi_{n m, k}^{(a, b)}\right]
\end{aligned}
$$

with $a \in\{x, y\}, b \in\{x, y\}, \bar{K}_{n m}$ is the average intercore coupling coefficient of the polarization directions given by $\bar{K}_{n m}=\left(K_{n m}^{(x)}+K_{n m}^{(y)}\right) / 2$ (Soeiro et al., 2017); $\omega$ is the angular frequency; $L$ is the MCF length; $\bar{\beta}_{m}$ and 
$\bar{\beta}_{n}$ are the average of the propagation constants over the two polarizations in cores $m$ and $n$ and are defined by, respectively, $\bar{\beta}_{m}=\left(\beta_{m}^{(x)}+\beta_{m}^{(y)}\right) / 2$ and $\bar{\beta}_{n}=\left(\beta_{n}^{(x)}\right.$ $\left.+\beta_{n}^{(y)}\right) / 2$ (Soeiro et al., 2017); $z_{k}$ is the longitudinal coordinate corresponding to the $k$-th phase matching point (PMP), with $N_{p}$ the number of PMPs (Cartaxo et al., 2016); $\phi_{n m, k}^{(a, b)}$ is the random phase shift (RPS) associated with the $k$-th PMP, and is modelled by an uniform distribution between 0 and $2 \pi$. The RPSs model the random variations of the bending radius, twist rate or other conditions in the MCF (Soeiro et al., 2017), (Cartaxo et al., 2016).

The dual-polarization DCM has been developed to keep the complexity and time of simulation at acceptable levels. In such model, the evolution of the ICXT impact on the system performance is evaluated in time fractions (named MCF realizations) much shorter than the ICXT decorrelation time. The MCF realizations are separated by time intervals longer than the decorrelation time of ICXT of the MCF. This means that, from MCF realization to MCF realization, the ICXT is uncorrelated and, within each MCF realization, is totally correlated. The different MCF realizations are obtained by generating randomly different sets of $N_{p}$ RPSs. In each iteration of the MC simulator, one MCF realization is generated. In each MCF realization, the bits of the interfering PAM2 signal with null extinction ratio transmitted in core $m$ are randomly generated. In the model shown in Fig. 2, the effect of CD is taken into account in both MCF cores $m$ and $n$. For equal powers at the output of the interfered and interfering cores, the ratio between the average crosstalk power and the average power of the signal, at the output of the tested core $n, X_{c}$, is related to the parameters of Eq. 5 by $X_{c}=N_{p}\left|\bar{K}_{n m}\right|^{2}$. The skew between the interfering core, $m$ and the tested core, $n$, is given by $S_{m n}=d_{m n} L$, where $d_{m n}$ is the walkoff between cores $m$ and $n$ and is related to Eq. 5 by $d_{m n}=\bar{\beta}_{1, m}-\bar{\beta}_{1, n}$, where $\bar{\beta}_{1, m}$ is the inverse of the group velocity of core $m$, and $\bar{\beta}_{1, n}$ is the inverse of the group velocity of core $n$.

\subsection{BER and OP Calculation}

In this subsection, the procedure for the BER and OP estimation in PAM4 systems with direct-detection and impaired by ICXT is described.

The BER is calculated by the semi-analytical method known as the exhaustive Gaussian approach applied to the PAM4 case, which is given by (Rebola and Cartaxo, 2000)

$$
\begin{gathered}
B E R=\frac{1}{4^{N_{b}}}\left\{\sum_{\substack{k=1 \\
a_{k}=0}}^{4^{N_{b}}} Q\left(\frac{F_{1}-i_{0, k}}{\sigma_{0, k}}\right)+\right. \\
\sum_{\substack{k=1 \\
a_{k}=1}}^{4^{N_{b}}}\left[Q\left(\frac{i_{1, k}-F_{1}}{\sigma_{1, k}}\right)+Q\left(\frac{F_{2}-i_{1, k}}{\sigma_{1, k}}\right)\right]+ \\
\sum_{\substack{4_{k}=1 \\
a_{k}=2}}^{N_{b}}\left[Q\left(\frac{i_{2, k}-F_{2}}{\sigma_{2, k}}\right)+Q\left(\frac{F_{3}-i_{2, k}}{\sigma_{2, k}}\right)\right]+ \\
\left.\sum_{\substack{k=1 \\
a_{k}=3}}^{4^{N_{b}}}\left(\frac{i_{3, k}-F_{3}}{\sigma_{3, k}}\right)\right\}
\end{gathered}
$$

where $i_{0, k}, i_{1, k}, i_{2, k}$ and $i_{3, k}$ correspond to the mean of the current at the input of the decision circuit for the symbols ' 0 ', '1', '2' and '3', respectively; and $\sigma_{0, k}, \sigma_{1, k}, \sigma_{2, k}$ and $\sigma_{3, k}$ correspond to the noise standard deviations of the same current for the different symbols (Rebola and Cartaxo, 2000). The means of the received currents are extracted from the simulated eye-pattern at the optimum sampling instants. The function $Q(x)$ is given by (Carlson and Crilly, 2010)

$$
Q(x)=\int_{x}^{\infty} \frac{1}{\sqrt{2 \pi}} e^{-\frac{\xi^{2}}{2}} d \xi
$$

In the simulation, the decision thresholds $F_{1}, F_{2}$ and $F_{3}$ are optimized by using the bisection method to minimize the BER (Srivastava and Dixit, 2012).

By considering a PAM4 sequence with $4^{N_{b}}$ symbols implies $4^{N_{b}}$ different levels of current at $4^{N_{b}}$ sampling time instants at the decision circuit input and, in this way, $4^{N_{b}}$ different contributions to the BER, as shown in Eq. 6. The effect of intersymbol interference from filtering and fiber dispersion is taken into account by the waveform distortion in the eyepattern at these $4^{N_{b}}$ sampling time instants. In Eq. 6, this effect is included in the mean currents $i_{0, k}, i_{1, k}$, $i_{2, k}$ and $i_{3, k}$. Also, the effect of ICXT on the interfered core is taken into account in these mean currents. The effect of noise is taken into account semianalytically in the standard deviations of the received symbols. In this case, as we consider thermal noise, $\sigma_{0, k}=\sigma_{1, k}=\sigma_{2, k}=\sigma_{3, k}=\sigma_{c}$, with the noise power given by

$$
\sigma_{c}^{2}=R_{\lambda}^{2} N E P^{2} B_{e, n}
$$

where $R_{\lambda}$ is the PIN responsivity, NEP is the noise equivalent power, defined as the the minimum optical power necessary to generate a photocurrent equal to the noise current of the photodetector and $B_{e, n}$ is the noise equivalent bandwidth of the electrical filter of the optical receiver. 
The OP is the probability of the system becoming unavailable when a target BER limit is reached (Rebola et al., 2019b), (Winzer and Foschini, 2011). In this work, the system is considered unavailable, i.e., it is in an outage period, when the BER in presence of ICXT surpasses the BER limit of $3.8 \times 10^{-3}$. In the simulation, the OP is estimated by (Rebola et al., 2019c)

$$
O P=\frac{N_{o}}{N_{r}}
$$

where $N_{o}$ is the number of occurrences of BER above the BER limit and $N_{r}$ is the number of simulated MCF realizations necessary to reach this number of occurrences. An acceptable outage probability in optical communications is typically lower than $10^{-4}$ (Winzer and Foschini, 2011), (Cvijetic et al., 2008).

\section{NUMERICAL RESULTS AND DISCUSSION}

In this section, the impact of ICXT on the performance of PAM4 signals transmission in short-haul links with direct-detection, considering the transmission of a PAM2 signal in the interfering core, is assessed. The simulation parameters used throughout this work to perform this study are presented in Table 1 .

Table 1: Simulation parameters and values.

\begin{tabular}{|c|c|}
\hline Simulation parameter & Value \\
\hline$R_{\lambda}$ & $1 \mathrm{~A} / \mathrm{W}$ \\
\hline$N E P$ & $10^{-12} \mathrm{~W} / \sqrt{\mathrm{Hz}}$ \\
\hline$B_{e, n}$ & $0.75 \times R_{s}$ \\
\hline Symbol rate & $R_{s}=56$ Gbaud \\
\hline Fiber length & $L=2 \mathrm{~km}$ \\
\hline $\begin{array}{c}\text { Number of generated } \\
\text { PAM4 symbols in each } \\
\text { MCF realization }\end{array}$ & $N=4^{4}$ \\
\hline Carrier wavelength & $\lambda=1550 \mathrm{~nm}$ \\
\hline $\begin{array}{c}\text { Chromatic } \\
\text { dispersion parameter }\end{array}$ & $D_{\lambda}=17 \mathrm{ps} /(\mathrm{nm} \cdot \mathrm{km})$ \\
\hline Number of PMPs & $N_{p}=1000$ \\
\hline $\begin{array}{c}\text { Skew-symbol rate } \\
\text { product }\end{array}$ & $\begin{array}{c}\left|S_{m n} \cdot R_{S}\right|=1000 \\
\left|S_{m n} \cdot R_{s}\right|=0.01\end{array}$ \\
\hline Reference BER & $3.8 \times 10^{-3}$ \\
\hline BER in absence of ICXT & $3.8 \times 10^{-5}$ \\
\hline
\end{tabular}

The number of PMPs is chosen to be high enough to characterize the ICXT mechanism rigorously (Cartaxo et al., 2016). Two different skews, $\left|S_{m n} \cdot R_{s}\right|=1000$ and $\left|S_{m n} \cdot R_{S}\right|=0.01$, are also chosen according to the conditions i) $\left|S_{m n} \cdot R_{S}\right| \gg 1$, where the symbol rate of the PAM signal is much higher than the ICXT decorrelation bandwidth (which is proportional to the inverse of the skew) (Alves and Cartaxo, 2019) and the ICXT creates amplitude levels that seem to exhibit a "noise" like-behavior (Rebola et al., 2019b), and ii) $\left|S_{m n} \cdot R_{S}\right| \ll 1$, where the symbol rate of the PAM signal is much lower than the ICXT decorrelation bandwidth and well-defined amplitude levels in the eye-patterns are created due to ICXT (Rebola et al., 2019a), (Alves and Cartaxo, 2019). Although intra-datacenters operate at a typical $1310 \mathrm{~nm}$ wavelength, the wavelength may need to be extended to $1550 \mathrm{~nm}$ to increase the transmission capacity. Furthermore, this wavelength allows to study the limitations induced by the interaction of CD with ICXT.

\subsection{BER in Each MCF Realization}

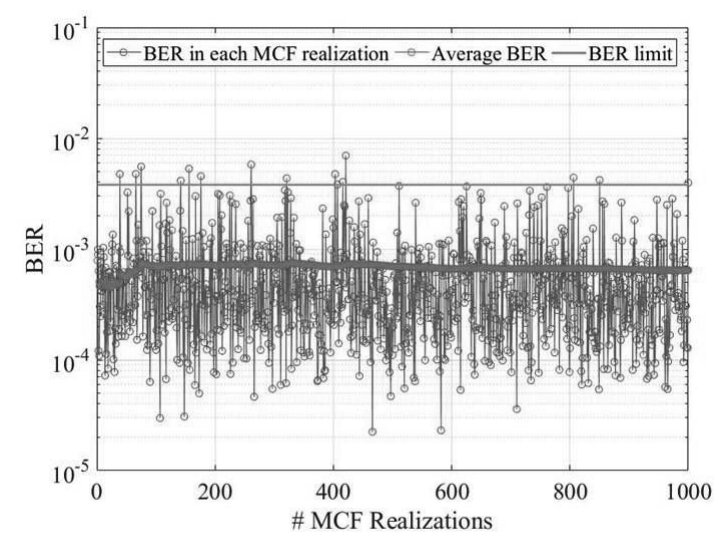

a)

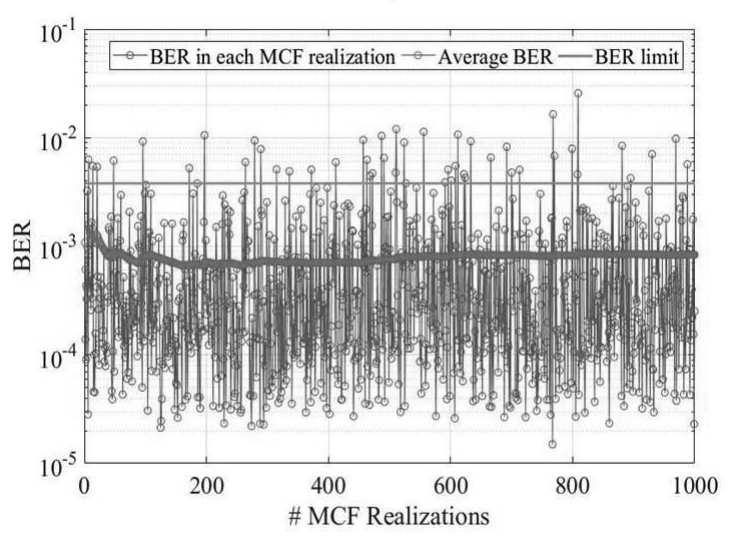

b)

Figure 4: BER in each MCF realization and average BER as a function of the number of MCF realizations, for $r=0$ and $X_{c}=-25 \mathrm{~dB}$, for a) $\left|S_{m n} \cdot R_{S}\right|=1000$ and b) $\left|S_{m n} \cdot R_{S}\right|=0.01$. The BER limit of $3.8 \times 10^{-3}$ is also depicted.

Fig. 4 show the BERs obtained in each MCF realization and the average BER as a function of the MCF realizations, for $r=0$ and $X_{c}=-25 \mathrm{~dB}$, for 
a) $\left|S_{m n} \cdot R_{s}\right|=1000$ and b) $\left|S_{m n} \cdot R_{S}\right|=0.01$. The average BER is computed in each MCF realization by averaging the BERs per realization calculated in the previous MCF realizations. In Figs. 4 a) and b), the average BER is stabilized at $6.5 \times 10^{-4}$ and $8.4 \times 10^{-4}$, respectively, after $1000 \mathrm{MCF}$ realizations. This number of MCF realizations is a conservative choice to achieve stabilized values of the average BER, as already concluded in (Rebola et al., 2019c). Comparatively to PAM2 transmission in the tested core (Rebola et al., 2019c), a much lower crosstalk level in PAM4 systems degrades much more the average BER in presence of ICXT than in PAM2 systems. In (Rebola et al., 2019c), for a crosstalk level of $X_{c}=-15 \mathrm{~dB}$ and for $\left|S_{m n} \cdot R_{b}\right|=10$ and $\left|S_{m n} \cdot R_{b}\right|=0.2$, after 1000 MCF realizations, the average BER is stabilized near $10^{-3}$. Fig. 4 shows that in a PAM4 system, this average BER is almost reached with a crosstalk level about $10 \mathrm{~dB}$ lower than the one predicted for the same average BER in a PAM2 transmission in the tested core. Thus, the performance of PAM4 transmission in the tested core is much more sensitive to ICXT than PAM2 signal transmission. Regarding the $\mathrm{OP}$, according to Fig. 4, we conclude that, with $\left|S_{m n} \cdot R_{s}\right|=0.01$, in 1000 MCF realizations, the BER limit is overcomed more times when compared with $\left|S_{m n} \cdot R_{s}\right|=1000$. In Fig. 4 a), the BER limit is exceeded about 15 times, i.e, there are 15 occurrences of the BER that lead to system outage. The OP, in this case, is approximately 0.015 . In Fig. 4 b), the BER limit is exceeded about 48 times, which indicates an approximated OP of 0.048. Similar conclusions regarding the number of MCF realizations to achieve an average BER and the lower tolerance of PAM4 signals to ICXT have been found for $r=0.1$.

\subsection{Eye-pattern Analysis}

Through the eye-pattern analysis, it is also possible to draw some conclusions regarding the impact of the ICXT induced by the transmission of PAM2 signals in the interfering core on the PAM4 signals performance in direct-detection links. Fig. 5 shows the eye-patterns at the decision circuit input for $\left|S_{m n} \cdot R_{s}\right|=1000, r=0$ and $X_{c}=-25 \mathrm{~dB}$, for a) the worst BER $\left(7 \times 10^{-3}\right)$ in each MCF realization and $b)$ the best BER $\left(2.2 \times 10^{-5}\right)$ in each MCF realization obtained in Fig. 4 a). Fig. 6 show the eyepatterns for $\left|S_{m n} \cdot R_{s}\right|=0.01, r=0$ and $X_{c}=-25 \mathrm{~dB}$, for a) the worst BER $\left(2.5 \times 10^{-2}\right)$ in each MCF realization and $b)$ the best BER $\left(1.5 \times 10^{-5}\right)$ in each MCF realization obtained in Fig. 4 b). From Figs. 5 and 6 , it can be observed that the eye-pattern in Fig. 6 a) shows a greater degradation due to ICXT when com-

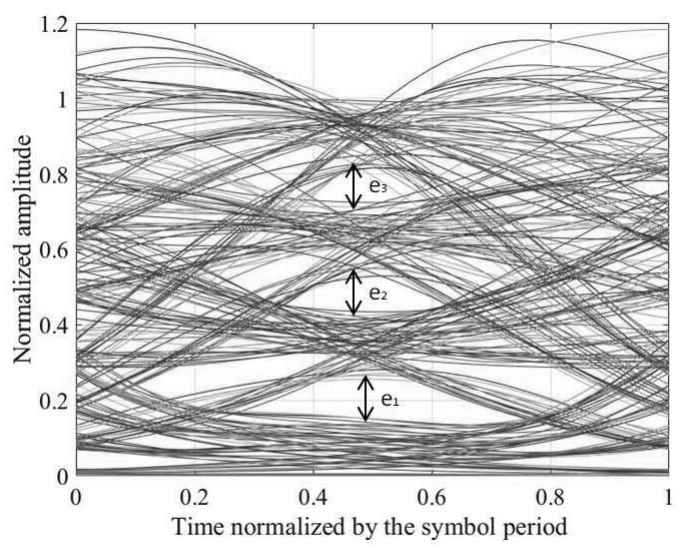

a)

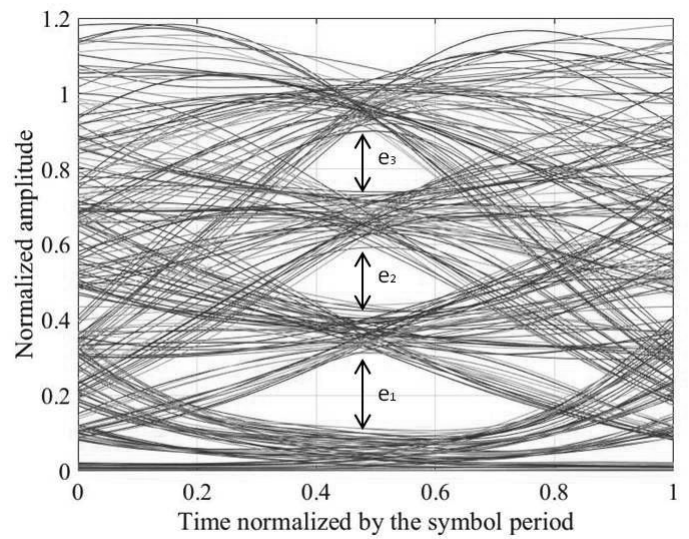

b)

Figure 5: Eye-patterns at the decision circuit input for $\left|S_{m n} \cdot R_{s}\right|=1000, r=0$ and $X_{c}=-25 \mathrm{~dB}$, for a) worst BER $\left(7 \times 10^{-3}\right)$ and b) best BER $\left(2.2 \times 10^{-5}\right)$ obtained in each MCF realization in Fig. 4 a).

pared with the eye-pattern in Fig. 5 a), especially in the superior eye concerning the symbols ' 2 ' and ' 3 ', since the eye opening is much lower. Figs. 5 b) and 6 b) present much similar eye openings which are much larger than the eye openings shown in Figs. 5 a) and 6 a) due to the lower influence of ICXT for the MCF realization that leads to the best BER. Also, Fig. 6 b) exhibits more "well-defined" amplitude levels due to ICXT than in the eye-pattern represented in Fig. 5 b), especially in the part of the eye where more symbol transitions occur. This effect happens because, for low $\left|S_{m n} \cdot R_{S}\right|$ (Fig. 6 b)), only one symbol in the interfering core is contributing to ICXT, while, for high $\left|S_{m n} \cdot R_{s}\right|$ (Fig. 5 b)), several symbols in the interfering core are contributing to ICXT. This effect has been already observed for PAM2 signal transmission in the tested core (Rebola et al., 2019c). The same studies were done for $r=0.1$, and similar results and conclusions were obtained. 


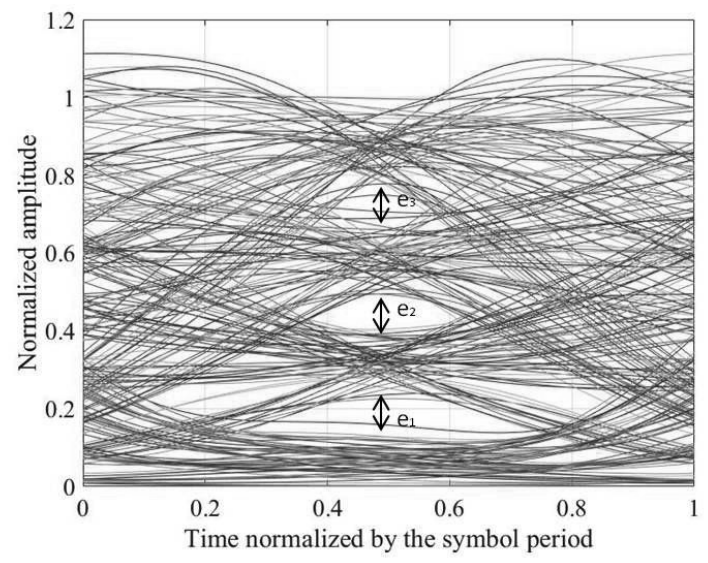

a)

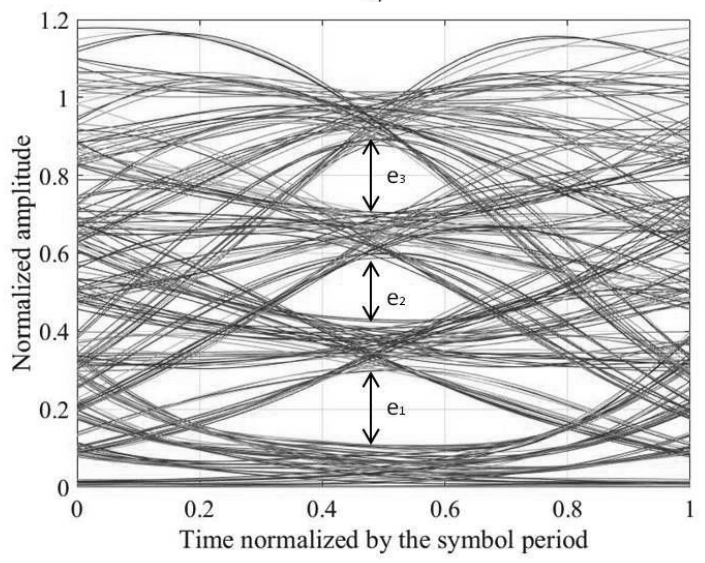

b)

Figure 6: Eye-patterns at the decision circuit input for $\left|S_{m n} \cdot R_{s}\right|=0.01, r=0$ and $X_{c}=-25 \mathrm{~dB}$, for a) worst BER $\left(2.5 \times 10^{-2}\right)$ and $\left.b\right)$ best BER $\left(1.5 \times 10^{-5}\right)$ obtained in each MCF realization in Fig. 4 b).

\subsection{Outage Probability}

The dependence of the OP estimation on the number of MCF realizations, for several $\left|S_{m n} \cdot R_{S}\right|$, extinction ratios and crosstalk levels is studied in the following. Fig. 7 shows the OP estimates as a function of the number of MCF realizations, for $r=0$, and a) $\left|S_{m n} \cdot R_{s}\right|=1000$ and $\left.X_{c}=-26 \mathrm{~dB} ; \mathrm{b}\right)$ $\left|S_{m n} \cdot R_{S}\right|=0.01$ and $X_{c}=-26 \mathrm{~dB}$ and c) $\left|S_{m n} \cdot R_{s}\right|=0.01$ and $X_{c}=-28.2 \mathrm{~dB}$. The BER is estimated in each MCF realization and the simulation is stopped when the number of occurrences of BER above the BER limit reaches 200, where we consider that the OP has been estimated with sufficient accuracy (Rebola et al., 2019c), (Rebola et al., 2019b). In Figs. 7 a) and c), the fluctuations of the OP estimates extend over a higher number of MCF realizations than in Fig. 7 b). According to Figs. 7 a) and b), it is possible to observe that, for the same crosstalk level and extinction ratio, a higher number of MCF realizations is needed to

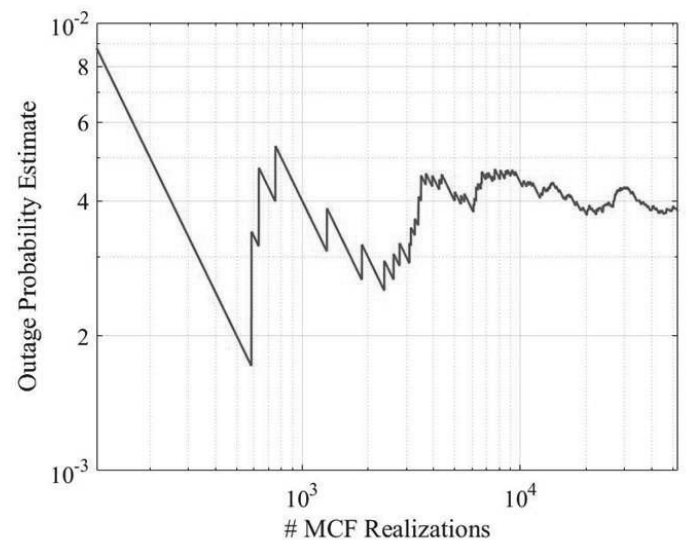

a)

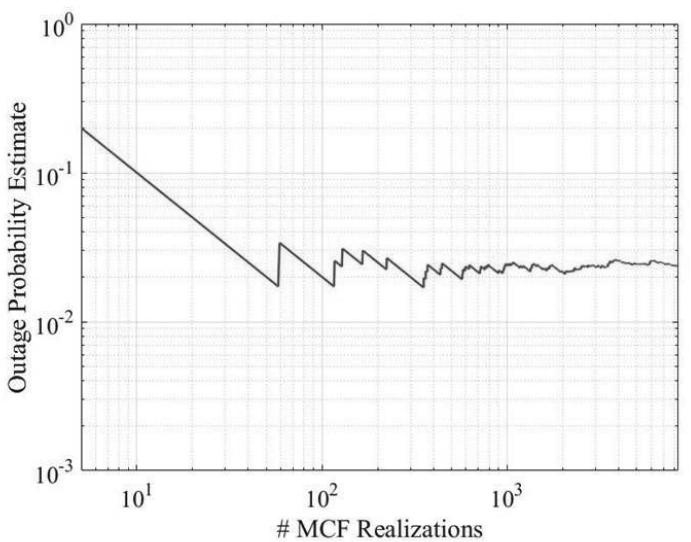

b)

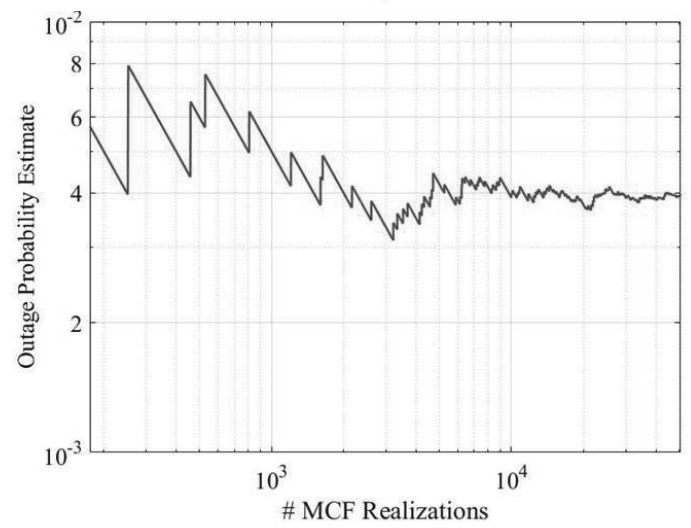

c)

Figure 7: Outage probability estimate as a function of the number of MCF realizations, for $r=0$ and a) $\left|S_{m n} \cdot R_{S}\right|=1000$ and $\left.X_{c}=-26 \mathrm{~dB} ; \mathrm{b}\right)\left|S_{m n} \cdot R_{S}\right|=0.01$ and $X_{c}=-26 \mathrm{~dB}$ and c) $\left|S_{m n} \cdot R_{S}\right|=0.01$ and $X_{c}=-28.2 \mathrm{~dB}$.

reach 200 occurrences of BER above the BER limit, with $\left|S_{m n} \cdot R_{S}\right|=1000$ than with $\left|S_{m n} \cdot R_{s}\right|=0.01$, since the OP of the system with the higher $\left|S_{m n} \cdot R_{S}\right|$, about $4 \times 10^{-3}$, is one order of magnitude lower than the one shown in Fig. 7 b) of about $2 \times 10^{-2}$. In Fig. 7 c), with $\left|S_{m n} \cdot R_{s}\right|=0.01$ and $X_{c}=-28.2 \mathrm{~dB}$, it is possible to 
observe that the number of MCF realizations is nearly the same as in Fig. 7 a), since the values of OP reached after 200 occurrences in both figures are very similar. Fig. 7 indicates that the number of MCF realizations necessary to estimate the OP with sufficient accuracy only depends on the order of magnitude of the OP as already hinted in other works (Rebola et al., 2019c), (Rebola et al., 2019b). In (Rebola et al., 2019c), the number of occurrences for which it is possible to obtain an OP of the optical communication system with very small fluctuations has been assessed for a PAM2 transmission in the tested core, and it has been concluded that 200 occurrences are more than enough to achieve this goal, which is in accordance with our results shown in Fig. 7. Thus, in this work, in all studies involving the OP estimation, $N_{o}=200$ is considered

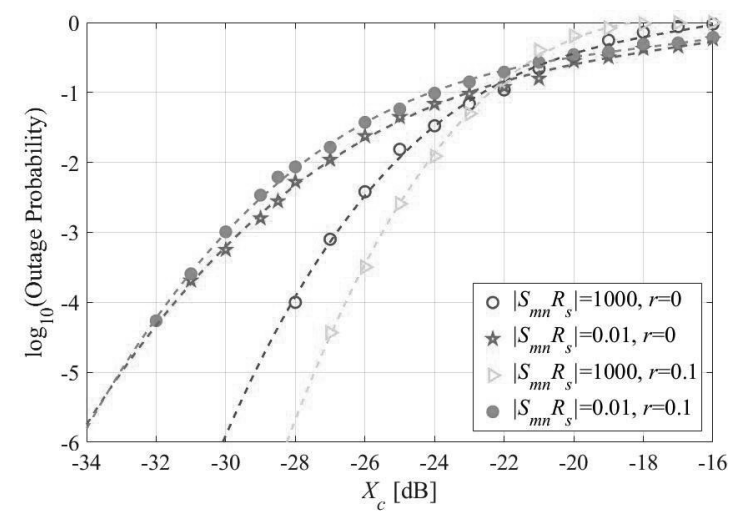

Figure 8: Outage probability as a function of $X_{c}$, for $\left|S_{m n} \cdot R_{S}\right|=1000$ and $r=0 ; \quad\left|S_{m n} \cdot R_{S}\right|=0.01$ and $r=0$; $\left|S_{m n} \cdot R_{S}\right|=1000$ and $r=0.1$ and $\left|S_{m n} \cdot R_{S}\right|=0.01$ and $r=0.1$. The dashed lines represent a cubic interpolation of the $\log _{10}$ of the outage probability.

Then, the OP in PAM4 transmission in the tested core, for a single interfering core with PAM2 transmission, for low and high $\left|S_{m n} \cdot R_{S}\right|$ and $r=0$ and $r=0.1$, has been obtained. Fig. 8 shows the OP obtained by simulation as a function of the ICXT level, for $\left|S_{m n} \cdot R_{s}\right|=1000$ and $r=0 ;\left|S_{m n} \cdot R_{s}\right|=$ 0.01 and $r=0 ;\left|S_{m n} \cdot R_{S}\right|=1000$ and $r=0.1$ and $\left|S_{m n} \cdot R_{s}\right|=0.01$ and $r=0.1$. For $X_{c} \geq-22 \mathrm{~dB}$, the system is unavailable with a very high OP above $10^{-1}$. Hence, for PAM4 systems impaired by ICXT induced by one interfering core, ICXT levels above this value are prohibitive. For lower crosstalk levels, with $\left|S_{m n} \cdot R_{s}\right|=1000$, for a BER limit of $10^{-3}$, for example, a higher crosstalk level is needed to achieve this OP than with $\left|S_{m n} \cdot R_{s}\right|=0.01$, regardless the extinction ratio. So, for high $\left|S_{m n} \cdot R_{s}\right|$, Fig. 8 indicates that the PAM4 system is more robust to outage than for lower $\left|S_{m n} \cdot R_{s}\right|$, as already concluded in (Rebola et al., 2019c), (Rebola et al., 2019b) for PAM2 sig- nalling. For $\left|S_{m n} \cdot R_{S}\right|=0.01$, comparing $r=0$ with $r=0.1$, the higher extinction ratio presents slightly higher OPs for the same crosstalk level. This suggests that, for low $\left|S_{m n} \cdot R_{s}\right|$, the influence of the extinction ratio on the OP is not much relevant. By comparing with PAM2 transmission in the tested core (Rebola et al., 2019c), a much lower ICXT level is needed to achieve the same outage probability with PAM4 signal transmission. For example, the crosstalk levels of $X_{c}=-18 \mathrm{~dB}$ and $X_{c}=-22 \mathrm{~dB}$ (Rebola et al., 2019c), lead to an outage probability of $10^{-3}$, in PAM2 systems, with $r=0$ and $\left|S_{m n} \cdot R_{b}\right|=10$ and $\left|S_{m n} \cdot R_{b}\right|=0.02$, respectively. As can be seen in Fig. 8, in PAM4 systems, the ICXT level that leads to this OP is much lower, $X_{c}=-26.9 \mathrm{~dB}$, for the higher $\left|S_{m n} \cdot R_{s}\right|$, which is $8.9 \mathrm{~dB}$ lower than in PAM2 systems, and $X_{c}=$ $-29.6 \mathrm{~dB}$, for the lower $\left|S_{m n} \cdot R_{S}\right|$, which is $7.6 \mathrm{~dB}$ lower than in PAM2 systems. Since the simulation takes a long time to reach lower OPs, around $10^{-4}$, the OP obtained for $X_{c}=-28 \mathrm{~dB},\left|S_{m n} \cdot R_{s}\right|=1000$ and $r=0$, has been obtained with only 100 occurrences and, for $X_{c}=-27 \mathrm{~dB},\left|S_{m n} \cdot R_{s}\right|=1000$ and $r=0.1$, the OP has been obtained with 47 occurrences. In this case, these simulations took more than one week to achieve 100 and 47 occurrences, respectively. As very low OPs $\left(\ll 10^{-4}\right)$ are computationally heavy to achieve using computer simulation, we have performed a cubic interpolation of $\log _{10}(\mathrm{OP})$ to achieve such lows OPs, similarly to what has been done in (Rebola et al., 2019c).

\section{CONCLUSIONS}

In this work, the impact of ICXT on the performance of PAM4 signals in short direct-detection links supported by MCFs has been studied through simulation. The influence of PAM2 signal transmission in the interfering core on the performance of PAM4 signal in the tested core has been assessed. We have shown that a number of MCF realizations of 1000 is more than enough to obtain a stabilized average BER of the PAM4 system. The impact of ICXT on the received PAM4 signals has been also studied through the eyepattern analysis. For both high and low $\left|S_{m n} \cdot R_{s}\right|$, and concerning the best achievable BER in one MCF realization, the eye openings are much similar to the one obtained in back-to-back configuration, due to the lower influence of ICXT for the MCF realization that leads to the best BER. Regarding the worst BER, for low $\left|S_{m n} \cdot R_{s}\right|$, the eye-patterns exhibit more "well-defined" amplitude levels due to ICXT. Also, the OP of the PAM4 system with direct-detection has been assessed. First, it was concluded that, for the 
same reference OP, high $\left|S_{m n} \cdot R_{s}\right|$ tolerates a higher ICXT level above about $2.3 \mathrm{~dB}$ than low $\left|S_{m n} \cdot R_{s}\right|$, independently of the extinction ratio. Regarding the extinction ratio, $r=0$ requires a lower ICXT level of about $1.4 \mathrm{~dB}$ than $r=0.1$, for the same reference OP. Comparing to PAM2 transmission in the tested core, a much lower ICXT level of about $8.9 \mathrm{~dB}$ and $7.6 \mathrm{~dB}$, for high and low $\left|S_{m n} \cdot R_{s}\right|$, respectively, is required to achieve the same OP in the PAM4 system. Hence, we have shown that short-haul links with direct-detection, typical of intra-datacenter connections, with PAM4 transmission are much less tolerant to the ICXT impairment than PAM2 systems.

\section{ACKNOWLEDGEMENTS}

This work is funded by FCT/MCTES through national funds and when applicable co-funded EU funds under the project UIDB/EEA/50008/2020 and MALEFICO project.

\section{REFERENCES}

Alves, T. and Cartaxo, A. (Feb. 1 2019). Decorrelation bandwidth of intercore crosstalk in weakly coupled multicore fibers with multiple interfering cores. Journal of Lightwave Technology, 37, no. 3:744-754.

Alves, T. and Cartaxo, A. (Nov. 1 2017). Intercore crosstalk in homogeneous multicore fibers: theoretical characterization of stochastic time evolution. Journal of Lightwave Technology, 35, no. 21:4613-4623.

Alves, T., Rebola, J., and Cartaxo, A. (San Diego, CA, USA, Mar. 2019). Outage probability due to intercore crosstalk in weakly-coupled MCF systems with OOK signaling. Optical Fiber Communications Conference and Exhibition (OFC), paper M2I.1.

Butler et al., D. (Feb. 15 2017). Space division multiplexing in short reach optical interconnects. Journal of Lightwave Technology, 35, no. 4.

Carlson, A. and Crilly, P. (New York, USA, 2010). Communication systems - An introduction to signals and noise in electrical communication. McGraw-Hill International Editions, $5^{\text {th }}$ edition.

Cartaxo, A. V. T., Luís, R. S., Puttnam, B. J., Hayashi, T., Awaji, Y., and Wada, N. (Sep. 17 2016). Dispersion impact on the crosstalk amplitude response of homogeneous multi-core fibers. IEEE Photonics Technology Letters, 28, no. 17:1858-1861.

Cisco (2018). Cisco global cloud index: forecast and methodology, 2016-2021. White paper, pages 1-46.

Cvijetic, N., Wilson, S., and Qian, D. (Jul. 15 2008). System outage probability due to PMD in high-speed optical OFDM transmission. Journal of Lightwave Technology, 26, no. 14:2118-2127.
Klaus et al., W. (Apr. 4 2017). Advanced space division multiplexing technologies for optical networks. Journal of Lightwave Technology, 9, no. 4:1-11.

Lv, W., Li, J., and Xu, K. (Shenzhen, China, Sep. 2016). Direct detection of PAM4 signals with receiver side digital signal processing for bandwidth-efficient shortreach optical transmissions. IEEE Optoelectronics Global Conference (OGC), pages 4-6.

Perin, J., Shastri, A., and Kahn, J. (2018). Data center links beyond 100Gbit/s per wavelength. Optical Fiber Technology, 44:69-85.

Rebola, J., Alves, T., and Cartaxo, A. (Angers, France, Jul. 2019a). Assessment of the combined effect of laser phase noise and intercore crosstalk on the outage probability of DD OOK systems. International Conference on Transparent Optical Networks (ICTON), papper We.D1.4.

Rebola, J. and Cartaxo, A. (Canada, 2000). Optimization of level spacing in quaternary optical communication systems. International Conference on Application of Photonic Technology (ICAPT), 4087:49-59.

Rebola, J., Cartaxo, A., Alves, T., and Marques, A. (Jul. 15 2019 b). Outage probability due to intercore crosstalk in dual-core fiber links with direct-detection. IEEE Photonics Technology Letters, 31, no. 14:1195-1198.

Rebola, J., Cartaxo, A., and Marques, A. (Jun. 2019c). 10 Gbps CPRI signals transmission impaired by intercore crosstalk in $5 \mathrm{G}$ network fronthauls with multicore fibers. Photonic Network Communications, 37, no. 3:409-420.

Telecommunications Standardization Section of ITU-T (2009). Optical fibres, cables and system, Handbooks on Standardization, ITU, Geneva.

Saitoh, K. and Shoichiro, M. (Jan. 1 2016). Multicore fiber technology. Journal of Lightwave Technology, 34, no. 1:55-66.

Soeiro, R. O. J., Alves, T. M. F., and Cartaxo, A. V. T. (Aug. $15,2017)$. Dual polarization discrete changes model of inter-core crosstalk in multi-core fibers. IEEE Photonics Technology Letters, 29, no. 16:1395-1398.

Srivastava, R. and Dixit, N. (2012). Numerical accuracy of bisection method. International Multidisciplinary Research Journal, 2, no. 1.

Winzer, P. and Foschini, G. (Aug. 15 2011). MIMO capacities and outage probabilities in spatially multiplexed optical transport systems. Optics Express, 19, no. 17:16680-16696.

Zhou, X., Urata, R., and Liu, H. (Mar. 2019). Beyond $1 \mathrm{~Tb} / \mathrm{s}$ datacenter interconnect technology: challenges and solutions. Optical Fiber Communications Conference and Exhibition (OFC), San Diego, CA, USA, paper Tu2F.5. 